\title{
Prevalence of Abnormal Glucose Regulation according to Different Diagnostic Criteria in Ischaemic Stroke without a History of Diabetes
}

\author{
Xinmiao Zhang, ${ }^{1,2,3,4}$ Qiuyan Shi, ${ }^{5}$ Huaguang Zheng, 1,2,3,4 \\ Qian Jia, ${ }^{1,2,3,4}$ Xingquan Zhao ${ }^{1}$, , ,2,3,4 Liping Liu ${ }^{10},{ }^{1,2,3,4}$ Chunxue Wang, ${ }^{1,2,3,4}$ Xia Meng, ${ }^{1,2,3,4}$ \\ Jing Jing, ${ }^{1,2,3,4}$ Yuesong Pan $\mathbb{D}^{1,2,3,4}$ Yilong Wang $\mathbb{D}^{1,},{ }^{1,2,3,4}$ and Yongjun Wang $\mathbb{D}^{1,2,3,4}$ \\ ${ }^{1}$ Department of Neurology, Beijing Tiantan Hospital, Capital Medical University, Beijing 100050, China \\ ${ }^{2}$ China National Clinical Research Center for Neurological Diseases, Beijing 100050, China \\ ${ }^{3}$ Center of Stroke, Beijing Institute for Brain Disorders, Beijing 100050, China \\ ${ }^{4}$ Beijing Key Laboratory of Translational Medicine for Cerebrovascular Disease, Beijing 100050, China \\ ${ }^{5}$ Department of Neurology, Affiliated Hospital, North China University of Science and Technology, Tangshan 063000, China
}

Correspondence should be addressed to Yilong Wang; yilong528@gmail.com and Yongjun Wang; yongjunwang1962@gmail.com

Received 19 September 2017; Revised 13 March 2018; Accepted 26 March 2018; Published 22 May 2018

Academic Editor: Vida Demarin

Copyright (c) 2018 Xinmiao Zhang et al. This is an open access article distributed under the Creative Commons Attribution License, which permits unrestricted use, distribution, and reproduction in any medium, provided the original work is properly cited.

\begin{abstract}
We aimed to investigate the prevalence and distribution of abnormal glucose regulation, including prediabetes and newly diagnosed diabetes, according to different criteria in ischaemic stroke patients without a history of diabetes. Data were derived from a representative cohort across China. Prediabetes was defined as fasting plasma glucose (FPG) 5.6-6.9 mmol/L or 2-hour oral glucose tolerance test (OGTT) $7.8-11.0 \mathrm{mmol} / \mathrm{L}$ or haemoglobin Alc (HbAlc) 5.7-6.4\%. Newly diagnosed diabetes was defined as FPG $\geq 7.0 \mathrm{mmol} / \mathrm{L}, 2 \mathrm{~h}$ OGTT $\geq 11.1 \mathrm{mmol} / \mathrm{L}$ or $\mathrm{HbAlc} \geq 6.5 \%$. Among 1251 ischaemic stroke patients, $471(37.5 \%)$ were detected as prediabetes and $539(43.1 \%)$ were detected as newly diagnosed diabetes. Prediabetes was present in 118 (9.4\%), 290 (23.2\%) and 314 (25.1\%) stroke patients, and newly diagnosed diabetes was present in 138 (11.0\%), 370 (29.6\%), and 365 (29.2\%) stroke patients, based on FPG, 2 h OGTT, and HbAlc criteria, respectively. Dependency on FPG alone would have missed $74.9 \%$ of patients in the prediabetes range and $74.4 \%$ of patients in the diabetes range. Our study demonstrated a high prevalence of prediabetes and diabetes in ischaemic stroke patients without a history of diabetes. OGTT and HbAlc helped detect the majority of prediabetes and newly diagnosed diabetes in ischaemic stroke patients.
\end{abstract}

\section{Introduction}

Diabetes is one of the most severe chronic diseases, which is also a contributor to the development of ischaemic stroke [13]. The prevalence of diabetes has increased rapidly in recent decades, especially in Asia, which was reported to be approximately $11.6 \%[4,5]$. Prediabetes, as an intermediate metabolic state between normal glucose regulation and diabetes, has an increasing incidence and high risk of developing diabetes [6]. Abnormal glucose regulation, including prediabetes and diabetes, often develops and interacts with ischaemic stroke. Abnormal glucose regulation doubles the risk of ischaemic stroke and worsens survival of patients with acute stroke [3, 7-9]. Previous studies showed that about one-third of ischaemic stroke patients had prediabetes, and $17.5 \%$ to $37.5 \%$ of stroke patients without a history of diabetes had unrecognized newly diagnosed diabetes [10-16]. In addition, abnormal glucose regulation has been proved to be associated with poor prognosis for ischaemic patients and has been widely used to predict the prognosis after an acute stroke as an important risk factor [17-22]. Hence, abnormal glucose regulation of stroke patients might be a therapeutic target for secondary prevention. Therefore, accurate diagnoses of abnormal glucose regulation is of great importance. 
In previous studies, abnormal glucose regulation was diagnosed mostly based on fasting plasma glucose (FPG) or random blood sugar on admission [10-22], a small proportion of which was diagnosed according to postload glucose ( $2 \mathrm{~h}$ oral glucose tolerance test [OGTT]) [14, 2225]. Recently, haemoglobin Alc (HbAlc) criteria have also been suggested as a diagnostic supplement by the American Diabetes Association and the World Health Organization [26, 27]. As reported, glucose-based and HbAlc-based criteria were frequently discordant regarding abnormal glucose regulation diagnosis [28]. Thus, using three diagnostic criteria combined (FPG, $2 \mathrm{~h}$ OGTT, and HbAlc) could increase the prevalence of abnormal glucose regulation, which may lead to a more standard secondary prevention and more favourable outcome. However, few studies, except one small European cohort [29], have investigated the prevalence and detailed distribution of prediabetes and newly diagnosed diabetes according to these three different diagnostic criteria in ischaemic stroke patients without a history of diabetes.

In our study, we used the Abnormal Glucose Regulation in Patients with Acute Stroke across China (ACROSS-China) registry to examine the prevalence and detailed distribution of prediabetes and newly diagnosed diabetes in ischaemic stroke patients without a history of diabetes according to different screening criteria (FPG, 2 h OGTT, and HbAlc).

\section{Material and Methods}

2.1. Study Participants. Ischaemic stroke patients without a history of diabetes in the survey on ACROSS-China were included in our study, details on the design and major results of which have been published previously [30]. Briefly, ACROSS-China is a well-designed, nationwide prospective cohort study that aimed to investigate the prevalence and distribution of abnormal glucose regulation in hospitalized patients (aged $\geq 18$ years) between 2008 and 2009 across China. ACROSS-China has excluded all patients receiving intravenous alimentation because they could not finish the OGTT test [22].

Acute ischaemic stroke was diagnosed according to the World Health Organization criteria [31] combined with brain computed tomography or magnetic resonance confirmation. Patients were diagnosed as acute ischaemic stroke when the following conditions were met: acute occurrence within 14 days of neurological deficit, focal or overall involvement of the nervous system; symptoms that lasted for >24 hours; and the exclusion of nonvascular causes (e.g., primary and metastatic neoplasms, postseizure paralysis, or head trauma) that led to brain function deficit and of intracerebral hemorrhage by computed tomography or magnetic resonance imaging. The definition of a history of diabetes was either a self-reported physician diagnosis of diabetes mellitus or hypoglycemic medications (e.g., insulin or sulfonylureas) before hospitalization [22]. The diagnostic criteria were consistent across all participating hospitals. Patients with insulin or oral hypoglycemic agents during hospitalization and with extremely high plasma glucose levels during hospitalization were excluded for the sake of patients' safety and lack of the OGTT results.
2.2. Data Collection. Patient baseline information was recorded at admission, including sex, age, height, weight, blood pressure, fasting glucose level, and triglyceride. Assessment of baseline vascular risk factors included history of stroke, hypertension, dyslipedaemia, atrial fibrillation, coronary heart disease, diabetes, and body mass index (BMI). The National Institutes of Health Stroke Scale (NIHSS) and Glasgow Coma Scale were used to evaluate the severity of neurologic impairment within 24 hours after admission and at discharge. The clinical subtypes of ischaemic stroke were classified according to the Oxfordshire Community Stroke Project criteria [32].

2.3. Assessment of Abnormal Glucose Regulation. Prediabetes was defined as FPG level of $5.6-6.9 \mathrm{mmol} / \mathrm{L}, 2 \mathrm{~h}$ OGTT level of $7.8-11.0 \mathrm{mmol} / \mathrm{L}$, or HbAlc level of 5.7-6.4\%. Newly diagnosed diabetes was defined as FPG $\geq 7.0 \mathrm{mmol} / \mathrm{L}, 2 \mathrm{~h}$ OGTT $\geq 11.1 \mathrm{mmol} / \mathrm{L}$, or HbAlc $\geq 6.5 \%$.

FPG, 2 h OGTT, and HbAlc levels were assessed in the study patients. A standard FPG and OGTT was performed in the ischaemic patients without previous diabetes during the morning hours (range: 07:00-11:00) on day $14 \pm 3$ after stroke onset or before discharge (if hospital stay $<14$ days) according to the World Health Organization criteria [33]. After overnight fasting (at least 8 hours), the patients drank $250 \mathrm{~mL}$ of a solution that included $75 \mathrm{~g}$ of glucose within 3 minutes. Immediately before administration of the drink and after 2 hours, venous blood samples were collected in sodium fluoride tubes to measure plasma glucose. Fasting and 2 h OGTT plasma glucose levels were measured ( $\mathrm{mmol} / \mathrm{L})$ via an automated glucose oxidation method. The first overnight fasting venous blood samples were drawn to measure the blood HbAlc levels following admission using high-performance liquid chromatographic analysis, which were aligned to the Diabetes Control and Complications Trial and the National Glycohemoglobin Standardization Program standards [34].

2.4. Statistical Analysis. We presented continuous variables as mean $\pm \mathrm{SD}$ or median with interquartile range and categorical variables as percentages. The demographic and clinical characteristics of the stroke patients with prediabetes and newly diagnosed diabetes were compared with patients with normal glucose regulation using the $\chi^{2}$ test for categorical variables and $t$-test or Wilcoxon rank-sum test for continuous variables, respectively. We also evaluated the prevalence of patients with prediabetes and newly diagnosed diabetes based on FPG, 2 h OGTT, and HbAlc criteria. The correlations between any two of the three diagnostic criteria were estimated. The data were analysed with SAS version 9.4 statistical software (SAS Institute Inc., Cary, NC, USA). All reported $P$ values were two-sided with $P<0.05$ being considered significant.

2.5. Ethical Approval. The study was approved by the Ethics Committee of Beijing Tiantan Hospital and all participating hospitals, in compliance with the Declaration of Helsinki. Written informed consent was obtained from all participants. All the experiments described were performed in accordance with the approved guidelines. 
TABLE 1: Baseline characteristics of patients in different states of glucose regulation.

\begin{tabular}{|c|c|c|c|c|}
\hline Variables & $\begin{array}{l}\text { Normal glucose } \\
\text { regulation } \\
(n=241) \\
\end{array}$ & $\begin{array}{c}\text { Prediabetes }{ }^{*} \\
\quad(n=471)\end{array}$ & $\begin{array}{c}\text { Newly diagnosed } \\
\text { diabetes }^{\ddagger} \\
(n=539)\end{array}$ & $P$ value \\
\hline Sex (men), $n(\%)$ & $171(71.0)$ & $304(64.8)$ & $314(58.6)$ & 0.003 \\
\hline Age, $y$, mean \pm SD & $58.3 \pm 12.6$ & $61.8 \pm 12.8$ & $64.2 \pm 11.9$ & $<0.001$ \\
\hline NIHSS score at admission, median (IQR) & $4(2-7)$ & $4(2-8)$ & $4(2-8)$ & 0.93 \\
\hline Body mass index, $\mathrm{kg} / \mathrm{m} 2$, mean $\pm \mathrm{SD}$ & $24.6 \pm 3.5$ & $24.7 \pm 3.9$ & $25.1 \pm 3.8$ & 0.07 \\
\hline Triglyceride at admission, $\mathrm{mmol} / \mathrm{L}$, mean $\pm \mathrm{SD}$ & $1.6 \pm 1.1$ & $1.7 \pm 1.0$ & $1.9 \pm 1.2$ & $<0.001$ \\
\hline $\mathrm{LDL}$ at admission, mmol/L, mean $\pm \mathrm{SD}$ & $2.9 \pm 0.9$ & $3.1 \pm 1.6$ & $3.2 \pm 1.5$ & 0.02 \\
\hline $\mathrm{HDL}$ at admission, $\mathrm{mmol} / \mathrm{L}$, mean $\pm \mathrm{SD}$ & $1.2 \pm 0.3$ & $1.2 \pm 0.3$ & $1.2 \pm 0.4$ & 0.20 \\
\hline History of hypertension, $n(\%)$ & $133(55.2)$ & $274(58.2)$ & $339(62.9)$ & 0.09 \\
\hline History of hyperlipidaemia, $n(\%)$ & $18(7.5)$ & $68(14.4)$ & $63(11.7)$ & 0.02 \\
\hline History of atrial fibrillation, $n(\%)$ & $9(3.7)$ & $37(7.9)$ & $25(4.6)$ & 0.03 \\
\hline History of coronary heart disease, $n(\%)$ & $23(9.5)$ & $61(13.0)$ & $70(13.0)$ & 0.35 \\
\hline Smoking, $n(\%)$ & & & & 0.58 \\
\hline Current smoker & $90(37.3)$ & $160(34.0)$ & $173(32.1)$ & \\
\hline Ever smoker & $19(7.9)$ & $49(10.4)$ & $54(10.0)$ & \\
\hline Nonsmoker & $132(54.8)$ & $262(55.6)$ & $312(57.9)$ & \\
\hline \multicolumn{5}{|l|}{ Medicine use during hospitalization, $n(\%)$} \\
\hline Oral hypoglycaemic drugs & $1(0.6)$ & $7(2.1)$ & $59(14.3)$ & $<0.001$ \\
\hline Insulin & $2(0.8)$ & $8(1.7)$ & $67(12.4)$ & $<0.001$ \\
\hline Antihypertensive drugs & $0(0.0)$ & $11(2.3)$ & $175(32.5)$ & $<0.001$ \\
\hline Diuretics & $5(2.1)$ & $13(2.8)$ & $11(2.0)$ & 0.72 \\
\hline B-blockers & $6(2.5)$ & $21(4.5)$ & $21(3.9)$ & 0.43 \\
\hline Statin & $107(44.4)$ & $253(53.7)$ & $277(51.4)$ & 0.06 \\
\hline Antiplatelet & $155(64.3)$ & $304(64.5)$ & $332(61.6)$ & 0.58 \\
\hline Anticoagulation & $12(5.0)$ & $27(5.7)$ & $36(6.7)$ & 0.62 \\
\hline Pulmonary infection, $n(\%)$ & $10(4.2)$ & $40(8.5)$ & $43(8.0)$ & 0.09 \\
\hline Urinary infection, $n(\%)$ & $11(4.6)$ & $22(4.7)$ & $17(3.2)$ & 0.42 \\
\hline TOAST subtypes, $n(\%)$ & & & & 0.02 \\
\hline Cardioembolism & $13(5.4)$ & $33(7.0)$ & $25(4.6)$ & \\
\hline Large artery atherosclerosis & $133(55.2)$ & $296(62.9)$ & $350(64.9)$ & \\
\hline Small artery occlusion & $73(30.3)$ & $122(25.9)$ & $140(26.0)$ & \\
\hline Other/undetermined & $10(4.2)$ & $7(1.5)$ & $5(0.9)$ & \\
\hline Undefined & $12(5.0)$ & $13(2.8)$ & $19(3.5)$ & \\
\hline
\end{tabular}

SD, standard deviation; IQR, interquartile range; NIHSS, National Institute of Health Stroke Scale; HDL, high-density lipoprotein; LDL, low-density lipoprotein; TOAST, trial of org 10172 in acute stroke treatment. ${ }^{*}$ Prediabetes was defined as fasting plasma glucose level of 5.6-6.9 mmol/L or 2-hour oral glucose tolerance test level of 7.8-11.0 mmol/L or haemoglobin Alc level of 5.7-6.4\%. ${ }^{\ddagger}$ Newly diagnosed diabetes was defined as fasting plasma glucose $\geq 7.0 \mathrm{mmol} / \mathrm{L}$ or 2 -hour oral glucose tolerance test $\geq 11.1 \mathrm{mmol} / \mathrm{L}$ or haemoglobin Alc $\geq 6.5 \%$.

\section{Results}

3.1. Study Participants and Characteristics. A total of 1251 ischaemic stroke patients without a history of diabetes were included in the final analysis. The patient selection has been previously reported [30]. The clinical characteristics of the included 1251 patients were shown in Tables 1 and 2. The average age was $62.2 \pm 12.6$ years and $789(63.3 \%)$ patients were men. For patients with prediabetes, they were older and more likely to have an increased level of LDL at admission, an increased frequency of a history of hyperlipidaemia and atrial fibrillation, and an increased possibility of being large arterial atherosclerosis, developing pulmonary infection, and receiving statin and antihypertensive drugs, compared with normal glucose regulation patients. For patients with newly diagnosed diabetes, they were older and less likely to be men. In addition, they were more likely to have increased levels of triglyceride and LDL at admission, an increased frequency of a history of hypertension, and an increased possibility of being large arterial atherosclerosis, developing pulmonary 


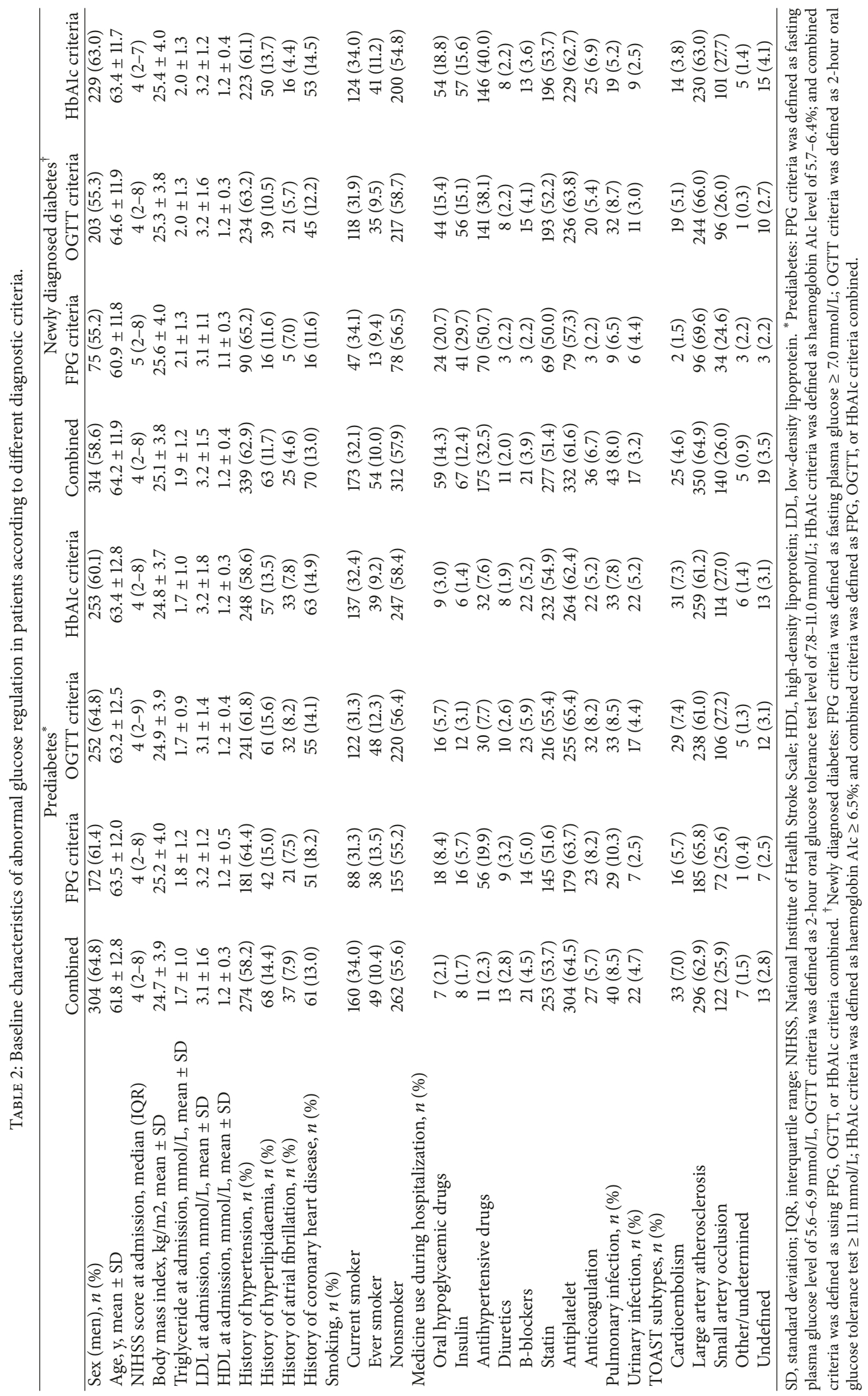




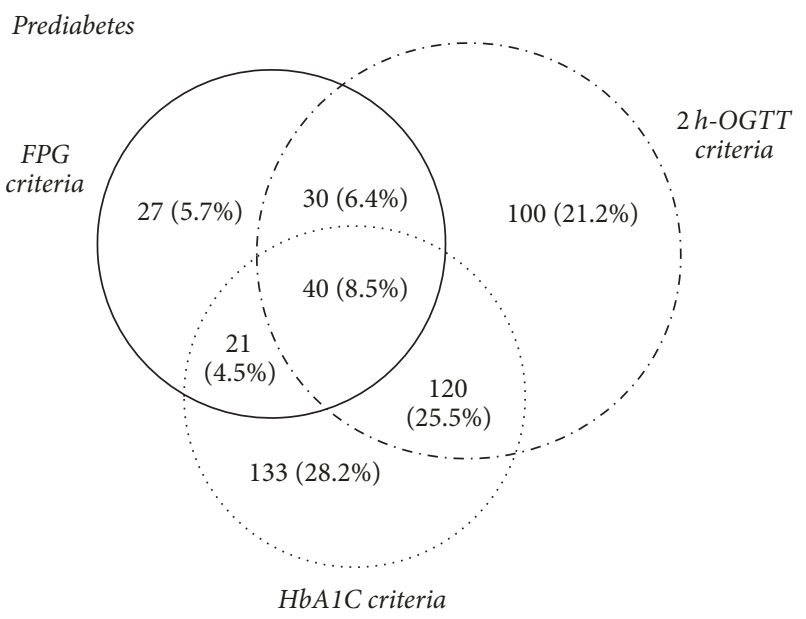

(a)

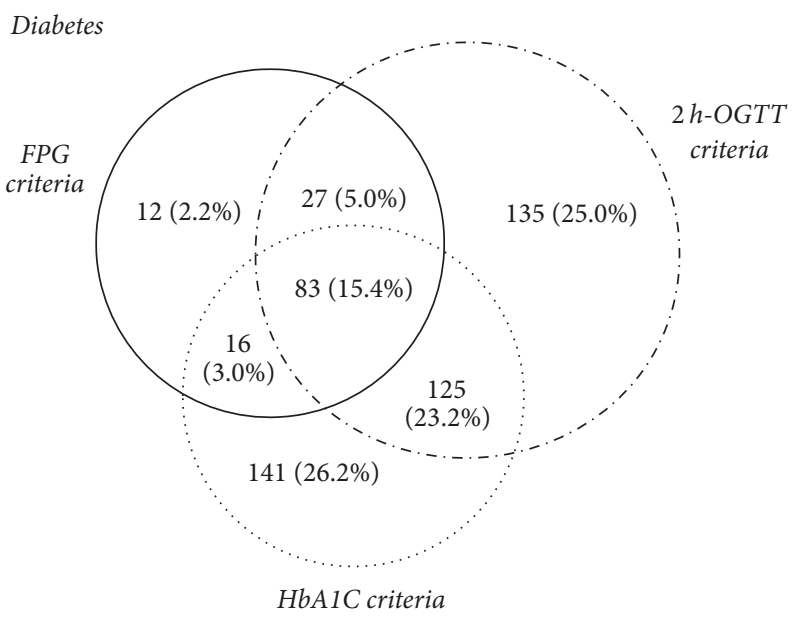

(b)

FIGURE 1: The prevalence and distribution according to different diagnostic criteria of prediabetes (a) and newly diagnosed diabetes (b) in ischaemic stroke patients without a history of diabetes. FPG criteria, fasting plasma glucose $\geq 7.0 \mathrm{mmol} / \mathrm{L}$; $2 \mathrm{~h}$ OGTT criteria, 2-hour oral glucose tolerance test $\geq 11.1 \mathrm{mmol} / \mathrm{L}$; HbAlc criteria, haemoglobin Alc $\geq 6.5 \%$.

infection, and receiving oral hypoglycaemic drugs, insulin, and antihypertensive drugs, compared with normal glucose regulation patients.

3.2. Prevalence and Distribution of Abnormal Glucose Regulation. The prevalence and detailed distribution of prediabetes and newly diagnosed diabetes identified by the three different diagnostic criteria were shown in Figure 1. 471 stroke patients (37.5\%) with prediabetes and 539 stroke patients (43.1\%) with newly diagnosed diabetes were detected based on all three detecting methods combined (FPG, 2 h OGTT, and HbAlc). Prediabetes was present in 118 (9.4\%), 290 (23.2\%), and $314(25.1 \%)$ of enrolled ischaemic stroke patients, based on FPG, 2-hour OGTT, and HbAlc criteria, respectively. Newly diagnosed diabetes was present in 138 (11.0\%), 370 (29.6\%), and 365 (29.2\%) of enrolled stroke patients, based on FPG, $2 \mathrm{~h}$ OGTT, and HbAlc criteria, respectively. Prediabetes and newly diagnosed diabetes were identified more frequently using 2 h OGTT $(23.2 \%, 29.6 \%)$ and HbAlc $(25.1 \%, 29.2 \%)$ than by FPG $(9.4 \%, 11.0 \%)$, respectively.

As shown in Figure 1, for patients with newly diagnosed diabetes, we detected $12(2.2 \%)$ with isolated elevated FPG, 135 (25.0\%) with isolated elevated $2 \mathrm{~h}$ OGTT, and 141 (26.2\%) with isolated elevated HbAlc. The rest of the patients with newly diagnosed diabetes $(46.6 \%)$ were diagnosed by the combined criteria. Using the traditional glucose-based criteria (FPG $\geq 7.0 \mathrm{mmol} / \mathrm{L}$, or OGTT $\geq 11.1 \mathrm{mmol} / \mathrm{L}$ ), 398 $(31.8 \%)$ patients were diagnosed newly diabetes, and the total percentage increased to $43.1 \%$ with the supplement of the HbAlc criteria ( $\mathrm{HbAlc} \geq 6.5 \%)$. These distribution characteristics of the three different diagnostic criteria were also seen in the prediabetes patients. Dependency on FPG alone would have missed $74.9 \%$ (353/471) of patients in the prediabetes range and $74.4 \%(401 / 539)$ of patients in the diabetes range.
The correlations between any two of the three diagnostic criteria were shown in Figure 2. Of the 832 patients with normal FPG level, 267 (32.1\%) had impaired glucose tolerance and $312(37.5 \%)$ had HbAlc levels in the prediabetes range. In addition, 147 (17.7\%) patients had $2 \mathrm{~h}$ OGTT levels in the diabetes range, and $143(17.2 \%)$ patients had HbAlc levels in the diabetes range. Furthermore, of the 281 patients with impaired fasting glucose, 113 (40.2\%) and 123 (43.8\%) had $2 \mathrm{~h}$ OGTT and HbAlc levels in the diabetes range, respectively.

\section{Discussion}

We investigated the prevalence and detailed distribution of prediabetes and newly diagnosed diabetes according to three different diagnostic criteria in ischaemic stroke patients without a history of diabetes. Our study demonstrated a high prevalence of prediabetes and newly diagnosed diabetes in ischaemic stroke patients. OGTT and HbAlc criteria helped detect the majority of prediabetes and unrecognized newly diabetes in ischaemic stroke patients.

Previous studies have demonstrated that the prevalence of prediabetes occurred in $31 \%$ to $38.5 \%$ of stroke patients and the prevalence of newly diagnosed diabetes in stroke patients was between 17.5 and $37.5 \%$ [10-16]. In our study, we found that prediabetes made up $37.6 \%$ of all ischaemic stroke patients without a history of diabetes based on three diagnosed criteria combined, as previous study reported. The newly diagnosed diabetes made up $43.1 \%$ of all ischaemic stroke patients without a history of diabetes, which was slightly more than that reported in previous study [29]. We infer that the most important reason was a high prevalence and low awareness of diabetes in China [4, 5]. A recent nationwide study showed that the prevalence of diabetes among Chinese aged 60 to 69 years was 25\%, and the proportion of those knowing of their diabetes was only $40 \%$ [35]. 


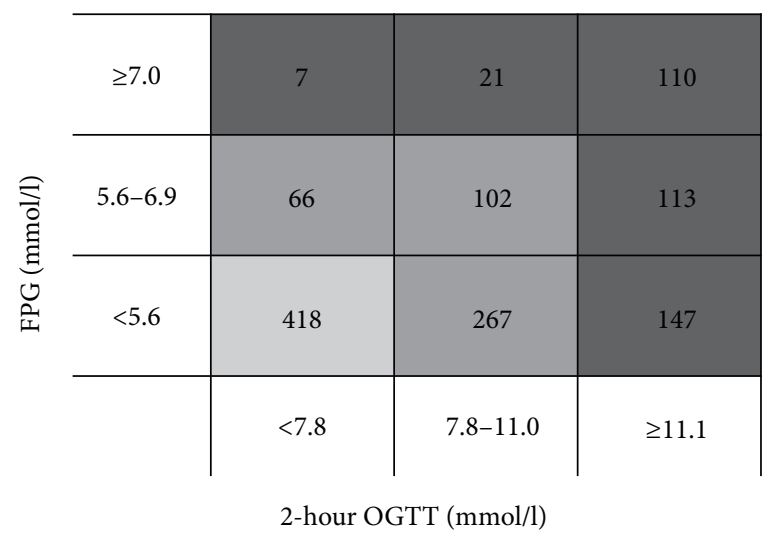

(a)

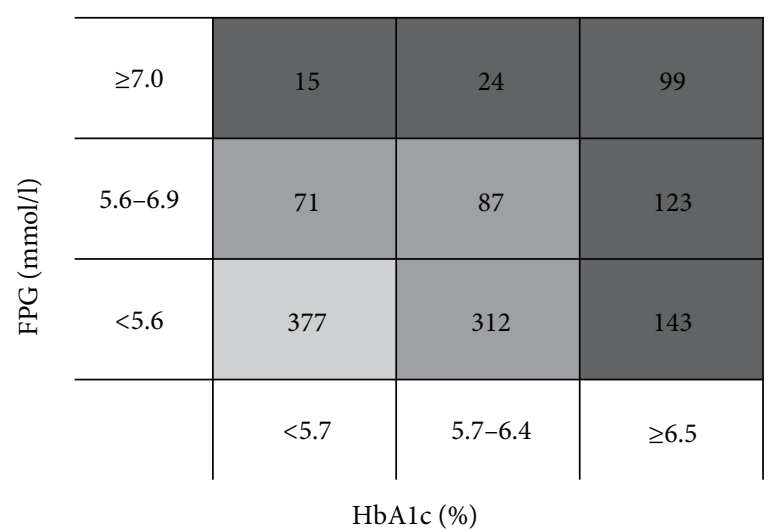

(b)

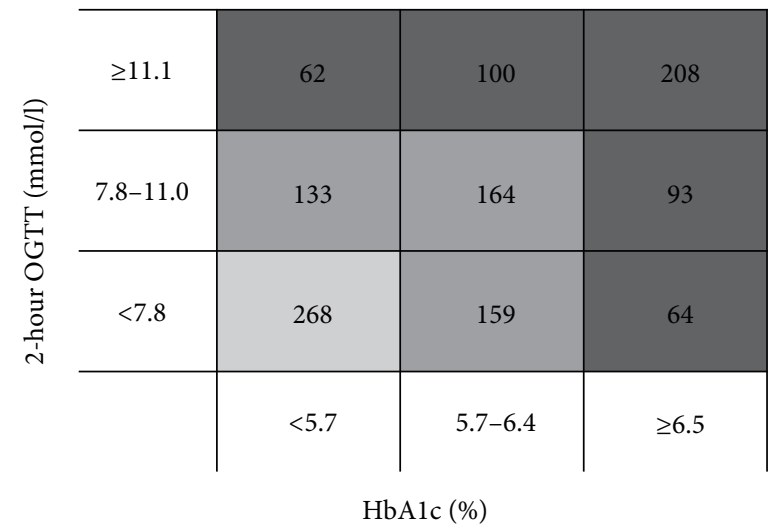

(c)

FIGURE 2: The figures $(\mathrm{a}-\mathrm{c}$ ) showed the correlative distribution between the different diagnostic methods. FPG, fasting plasma glucose; OGTT, oral glucose tolerance test; HbA1c, haemoglobin Alc.

The glucose-based (FPG and $2 \mathrm{~h}$ OGTT) and HbAlcbased criteria were frequently discordant regarding abnormal glucose regulation diagnosis [36]. Therefore, combining all three diagnostic criteria (FPG, 2 h OGTT, and HbAlc) may help detect more prediabetes and unrecognized newly diagnosed diabetes patients. Few studies, except for a European study [29], have investigated the prevalence and detailed distribution of prediabetes and newly diabetes diagnosed with these three criteria methods in ischaemic stroke patients. In this European study, however, the sample size was limited $(n=374)$ and the patients were confined to European stroke patients. In our study, prediabetes was present in 118 (9.4\%) of the ischaemic stroke patients using the FPG criteria alone. By adding the $2 \mathrm{~h}$ OGTT criteria, the percentage increased to $27.0 \%$. After adding the HbAlc criteria, the corresponding percentage reached $37.6 \%$. For patients with newly diagnosed diabetes, the corresponding proportions based on the different criteria were $11.0 \%, 31.8 \%$, and $43.1 \%$, respectively. Therefore, using the combined diagnostic criteria, there were many more abnormal glucose regulation patients detected after adding patients with single high OGTT and HbAlc levels.

The OGTT and HbAlc criteria played an indispensable role in accurately diagnosing abnormal glucose regulation in our study. The majority of ischaemic stroke patients with prediabetes (94.3\%) and newly diagnosed diabetes (97.8\%) were detected based on 2 h OGTT or HbAlc criteria. Firstly, it was common for Asian population to be diagnosed prediabetes and diabetes by $2 \mathrm{~h}$ OGTT which considered normal according to FPG [37]. Previous studies [14, 23] have used OGTT to detect prediabetes and newly diagnosed diabetes in stroke patients and found a high prevalence. In our study, we also observed similar results in diagnosing prediabetes $(61.6 \%, 290 / 471)$ and newly diagnosed diabetes (68.6\%, $370 / 539)$ based on the $2 \mathrm{~h}$ OGTT criteria. Dependency on FPG levels alone would have missed $49.8 \%$ (414/832) of patients with an abnormal $2 \mathrm{~h}$ OGTT. In addition, OGTT test has been proved to be a better predictor of vascular events and poor prognosis than FPG levels [24, 25]. All of these studies indicate the importance of performing OGTT to detect abnormal glucose regulation among stroke patients without a previous history of diabetes. Secondly, HbAlc, which has recently been proposed as an alternative to diagnose diabetes [26], has helped avoiding omitting plenty undiagnosed prediabetes and diabetes patients. In our study, prediabetes was present in $25.1 \%(314 / 1251)$ and newly diagnosed diabetes was present in $29.1 \%(365 / 1251)$ of ischaemic stroke patients based on HbAlc levels, which identified twice as many as 
the FPG criteria alone. In addition, high HbAlc levels may illustrate persistent hyperglycaemia, which has been proved to be associated with worse functional outcomes [38, 39]. Furthermore, HbAlc levels can be used in stroke patients with dysphagia, which adds to the importance of HbAlc in stroke patients.

The early recognition of abnormal glucose regulation in ischaemic stroke patients is significant, as vascular complications are preventable through strict glycaemic control $[40,41]$. As the indispensable components to diagnose prediabetes and newly diagnosed diabetes, OGTT and HbAlc should be attached importance equally as FPG did. Using single indicator (FPG, 2 h OGTT, or HbAlc) as the diagnostic criteria of prediabetes and newly diagnosed diabetes may lead to a large proportion of missed diagnoses. Overlooking OGTT and HbAlc examination may yield an important fraction of patients with abnormal glucose regulation. Therefore, our findings recommend the significance of integrated glucose examination at admission of stroke patients without a history of diabetes, which includes FPG, OGTT, and HbAlc, especially in the Chinese population.

Our study has several limitations. First, some ischaemic stroke patients were excluded because of the lack of OGTT (including dysphagia) and HbAlc data in the hospital, which may have led to bias. However, no significant differences in sex, vascular risk factors, or medical history were identified between the included and excluded patients. Second, all FPG and OGTT were tested on days $14 \pm 3$ after the stroke onset or before discharge (if hospital stay was $<14$ days) according to previous studies. However, it is difficult to avoid mixed patients with stress hyperglycemia, which could lead to bias. Third, the OGTT and HbAlc tests were not repeated according to the advice of the American Diabetes Association and newly diagnosed diabetes may be overestimated in our study. Finally, patients treated with insulin or oral hypoglycemic agents probably had diabetes even though FPG or 2-hour OGTT levels were within the normal range. In these patients, relationship between FPG or 2-hour OGTT and HbAlc could lead to a bias by the treatments.

\section{Conclusion}

Our findings demonstrated a high prevalence of prediabetes and newly diagnosed diabetes in ischaemic stroke patients without a history of diabetes. $2 \mathrm{~h}$ OGTT and HbAlc criteria helped detect the majority of prediabetes and unrecognized newly diabetes in ischaemic stroke patients.

\section{Conflicts of Interest}

The authors declare that they have no conflicts of interest.

\section{Authors' Contributions}

Xinmiao Zhang and Qiuyan Shi contributed equally to this work.

\section{Acknowledgments}

The study was supported by grants from National Key Technology Research and Development Program of the Ministry of Science and Technology of the People's Republic of China (2015BAI12B04 and 2015BAI12B02) and a grant from the Beijing Municipal Administration of Hospitals' Mission Plan (SML20150502).

\section{References}

[1] R. J. Jarrett, P. McCartney, and H. Keen, “The Bedford Survey: Ten year mortality rates in newly diagnosed diabetics, borderline diabetics and normoglycaemic controls and risk indices for coronary heart disease in borderline diabetics," Diabetologia, vol. 22, no. 2, pp. 79-84, 1982.

[2] H. King and M. Rewers, "Global estimates for prevalence of diabetes mellitus and impaired glucose tolerance in adults," Diabetes Care, vol. 16, no. 1, pp. 157-177, 1993.

[3] C. Emerging Risk Factors, N. Sarwar, P. Gao et al., "Diabetes mellitus, fasting blood glucose concentration, and risk of vascular disease: a collaborative meta-analysis of 102 prospective studies," The Lancet, vol. 375, no. 9733, pp. 2215-2222, 2010.

[4] Y. Xu, L. Wang, J. He et al., "Prevalence and control of diabetes in Chinese adults," Journal of the American Medical Association, vol. 310, no. 9, pp. 948-958, 2013.

[5] W. Yang, J. Lu, J. Weng et al., "Prevalence of diabetes among men and women in China," The New England Journal of Medicine, vol. 362, no. 12, pp. 1090-1101, 2010.

[6] E. S. Ford, G. Zhao, and C. Li, "Pre-diabetes and the risk for cardiovascular disease: a systematic review of the evidence," Journal of the American College of Cardiology, vol. 55, no. 13, pp. 1310-1317, 2010.

[7] L. B. Goldstein, R. Adams, K. Becker et al., "Primary prevention of ischemic stroke: A statement for healthcare professionals from the stroke council of the American Heart Association," Circulation, vol. 103, no. 1, pp. 163-182, 2001.

[8] M. J. A. Luitse, G. J. Biessels, G. E. H. M. Rutten, and L. J. Kappelle, "Diabetes, hyperglycaemia, and acute ischaemic stroke," The Lancet Neurology, vol. 11, no. 3, pp. 261-271, 2012.

[9] S. E. Vermeer, W. Sandee, A. Algra, P. J. Koudstaal, L. J. Kappelle, and D. W. J. Dippel, "Impaired glucose tolerance increases stroke risk in nondiabetic patients with transient ischemic attack or minor ischemic stroke," Stroke, vol. 37, no. 6, pp. 14131417, 2006.

[10] C. S. Gray, R. Taylor, J. M. French et al., "The Prognostic Value of Stress Hyperglycaemia and Previously Unrecognized Diabetes in Acute Stroke," Diabetic Medicine, vol. 4, no. 3, pp. 237-240, 1987.

[11] L. Kiers, S. M. Davis, R. Larkins et al., "Stroke topography and outcome in relation to hyperglycaemia and diabetes," Journal of Neurology, Neurosurgery \& Psychiatry, vol. 55, no. 4, pp. 263270, 1992.

[12] S. M. Oppenheimer, B. I. Hoffbrand, G. A. Oswald, and J. S. Yudkin, "Diabetes mellitus and early mortality from stroke," British Medical Journal (Clinical Research ed.), vol. 291, no. 6501, pp. 1014-1015, 1985.

[13] M. C. Riddle and J. Hart, "Hyperglycemia, recognized and unrecognized, as a risk factor for stroke and transient ischemic attacks," Stroke, vol. 13, no. 3, pp. 356-359, 1982. 
[14] C. S. Gray, J. F. Scott, J. M. French, K. G. M. M. Alberti, and J. E. O'Connell, "Prevalence and prediction of unrecognised diabetes mellitus and impaired glucose tolerance following acute stroke," Age and Ageing, vol. 33, no. 1, pp. 71-77, 2004.

[15] K. Matz, K. Keresztes, C. Tatschl et al., "Disorders of glucose metabolism in acute stroke patients: An underrecognized problem," Diabetes Care, vol. 29, no. 4, pp. 792-797, 2006.

[16] F. Vancheri, M. Curcio, A. Burgio et al., "Impaired glucose metabolism in patients with acute stroke and no previous diagnosis of diabetes mellitus," QJM: Monthly Journal of the Association of Physicians, vol. 98, no. 12, pp. 871-878, 2005.

[17] S. C. Johnston, P. M. Rothwell, M. N. Nguyen-Huynh et al., "Validation and refinement of scores to predict very early stroke risk after transient ischaemic attack," The Lancet, vol. 369, no. 9558, pp. 283-292, 2007.

[18] E. E. Smith, N. Shobha, D. Dai et al., "Risk score for in-hospital ischemic stroke mortality derived and validated within the get with the guidelines-stroke program," Circulation, vol. 122, no. 15, pp. 1496-1504, 2010.

[19] C. Weimar, H.-C. Diener, M. J. Alberts et al., "The Essen Stroke Risk Score predicts recurrent cardiovascular events A validation within the REduction of Atherothrombosis for Continued Health (REACH) registry," Stroke, vol. 40, no. 2, pp. 350-354, 2009.

[20] M. Lee, J. L. Saver, K.-S. Hong, S. Song, K.-H. Chang, and B. Ovbiagele, "Effect of pre-diabetes on future risk of stroke: Metaanalysis," BMJ, vol. 344, no. 7861, Article ID e3564, 2012.

[21] Q. Jia, X. Zhao, C. Wang et al., "Diabetes and poor outcomes within 6 months after acute ischemic stroke: The China national stroke registry," Stroke, vol. 42, no. 10, pp. 2758-2762, 2011.

[22] Q. Jia, G. Liu, H. Zheng et al., "Impaired glucose regulation predicted 1-year mortality of chinese patients with ischemic stroke: Data from abnormal glucose regulation in patients with acute stroke across China," Stroke, vol. 45, no. 5, pp. 1498-1500, 2014.

[23] F. M. Ivey, A. S. Ryan, C. E. Hafer-Macko et al., "High prevalence of abnormal glucose metabolism and poor sensitivity of fasting plasma glucose in the chronic phase of stroke," Cerebrovascular Disease, vol. 22, no. 5-6, pp. 368-371, 2006.

[24] N. L. Smith, J. I. Barzilay, D. Shaffer et al., "Fasting and 2hour postchallenge serum glucose measures and risk of incident cardiovascular events in the elderly: the Cardiovascular Health Study," JAMA Internal Medicine, vol. 162, no. 2, pp. 209-216, 2002.

[25] T. S. Temelkova-Kurktschiev, C. Koehler, E. Henkel, W. Leonhardt, K. Fuecker, and M. Hanefeld, "Postchallenge plasma glucose and glycemic spikes are more strongly associated with atherosclerosis than fasting glucose or $\mathrm{HbA}(1 \mathrm{c})$ level," Diabetes Care, vol. 23, no. 12, pp. 1830-1834, 2000.

[26] The International Expert Committee, "International Expert Committee report on the role of the A1C assay in the diagnosis of diabetes," Diabetes Care, vol. 32, no. 7, pp. 1327-1334, 2009.

[27] WHO Guidelines Approved by the Guidelines Review Committee, Use of glycated haemoglobin (HbA1C) in the diagnosis of diabetes mellitus: abbreviated report of a WHO consultation, World Health Organization, Geneva, 2011.

[28] L. Chen, D. J. Magliano, and P. Z. Zimmet, "The worldwide epidemiology of type 2 diabetes mellitus-present and future perspectives," Nature Reviews Endocrinology, vol. 8, no. 4, pp. 228-236, 2012.

[29] S. Fonville, A. A. M. Zandbergen, S. E. Vermeer, D. W. J. Dippel, P. J. Koudstaal, and H. M. Den Hertog, "Prevalence of prediabetes and newly diagnosed diabetes in patients with a transient ischemic attack or stroke," Cerebrovascular Disease, vol. 36, no. 4, pp. 283-289, 2013.

[30] J. Jing, Y. Pan, X. Zhao et al., "Prognosis of Ischemic Stroke with Newly Diagnosed Diabetes Mellitus According to Hemoglobin Alc Criteria in Chinese Population," Stroke, vol. 47, no. 8, pp. 2038-2044, 2016.

[31] WHO, "WHO Task Force on Stroke and other Cerebrovascular Disorders: stroke Recommendations on stroke prevention, diagnosis and therapy," Stroke, vol. 20, no. 10, pp. 1407-1431, 1989.

[32] H. P. Adams Jr., B. H. Bendixen, L. J. Kappelle et al., "Classification of subtype of acute ischemic stroke: definitions for use in a multicenter clinical trial," Stroke, vol. 24, no. 1, pp. 35-41, 1993.

[33] K. G. M. M. Alberti and P. Z. Zimmet, "Definition, diagnosis and classification of diabetes mellitus and its complications. Part 1: diagnosis and classification of diabetes mellitus. Provisional report of a WHO consultation," Diabetic Medicine, vol. 15, no. 7, pp. 539-553, 1998.

[34] R. R. Little, C. L. Rohlfing, H.-M. Wiedmeyer, G. L. Myers, D. B. Sacks, and D. E. Goldstein, "The National Glycohemoglobin Standardization Program: a five-year progress report," Clinical Chemistry, vol. 47, no. 11, pp. 1985-1992, 2001.

[35] G. Ning and Z. Bloomgarden, "Diabetes in China: Prevalence, diagnosis, and control," Journal of Diabetes, vol. 5, no. 4, pp. 372372, 2013.

[36] L. Chen, D. J. Magliano, and P. Z. Zimmet, “The worldwide epidemiology of type 2 diabetes mellitus-present and future perspectives," Nature Reviews Endocrinology, vol. 8, no. 4, pp. 228-236, 2011.

[37] Q. Qiao, K. Pyörälä, M. Pyörälä et al., “Two-hour glucose is a better risk predictor for incident coronary heart disease and cardiovascular mortality than fasting glucose," European Heart Journal, vol. 23, no. 16, pp. 1267-1275, 2002.

[38] T. A. Baird, M. W. Parsons, T. Phan et al., "Persistent poststroke hyperglycemia is independently associated with infarct expansion and worse clinical outcome," Stroke, vol. 34, no. 9, pp. 22082214, 2003.

[39] M. Kamouchi, T. Matsuki, J. Hata et al., "Prestroke glycemic control is associated with the functional outcome in acute ischemic stroke: the fukuoka stroke registry," Stroke, vol. 42, no. 10, pp. 2788-2794, 2011.

[40] DECODE Study Group on behalf of the European Diabetes Epidemiology Study Group, "Will new diagnostic criteria for diabetes mellitus change phenotype of patients with diabetes? Reanalysis of European epidemiological data," British Medical Journal, vol. 317, no. 7155, pp. 371-375, 1998.

[41] "The effect of intensive treatment of diabetes on the development and progression of long-term complications in insulindependent diabetes mellitus. The Diabetes Control and Complications Trial Research Group," The New England Journal of Medicine, vol. 329, no. 14, pp. 977-986, 1993. 


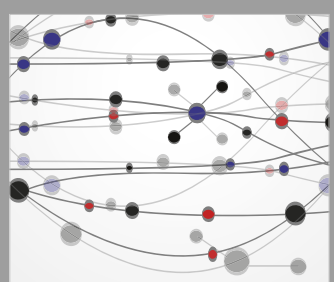

The Scientific World Journal
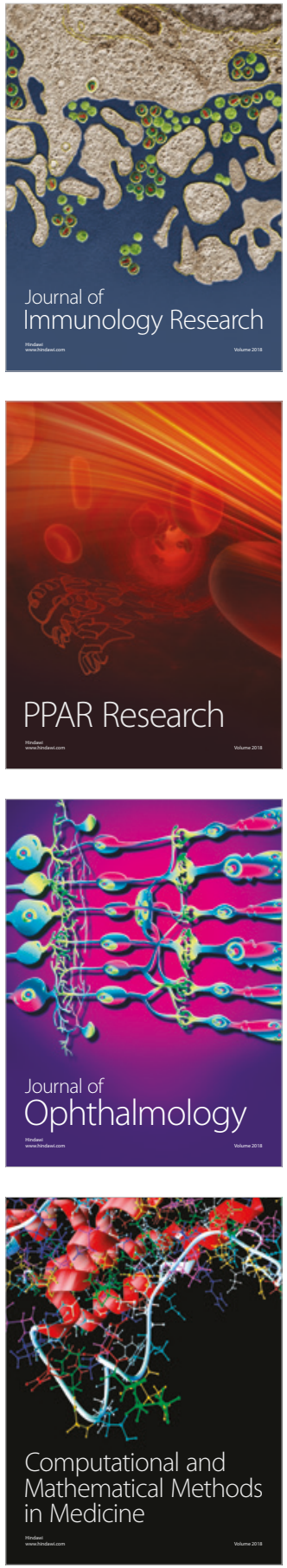

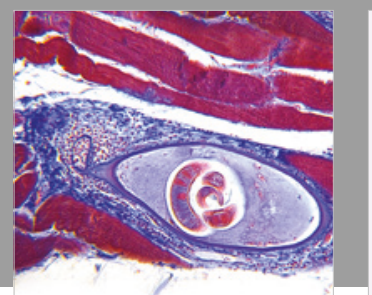

Gastroenterology Research and Practice

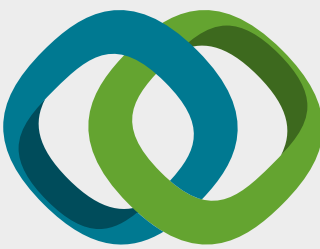

\section{Hindawi}

Submit your manuscripts at

www.hindawi.com
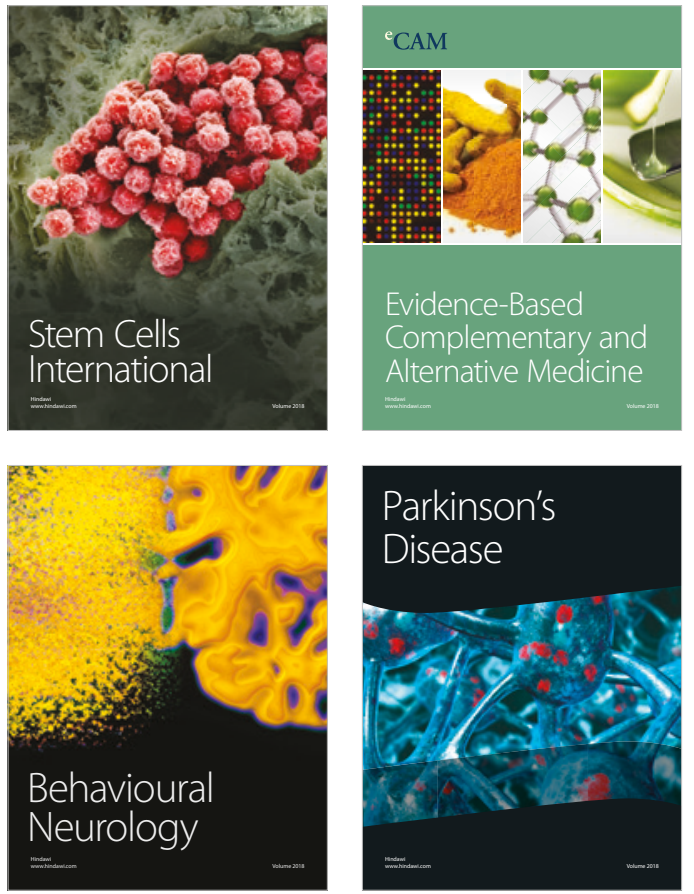

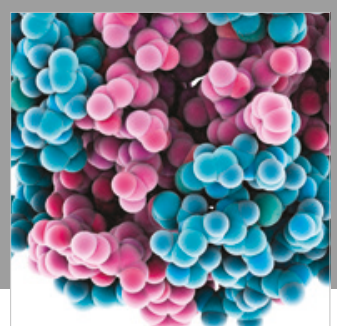

ournal of

Diabetes Research

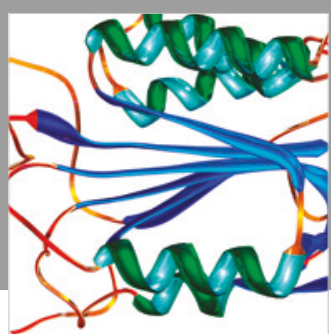

Disease Markers
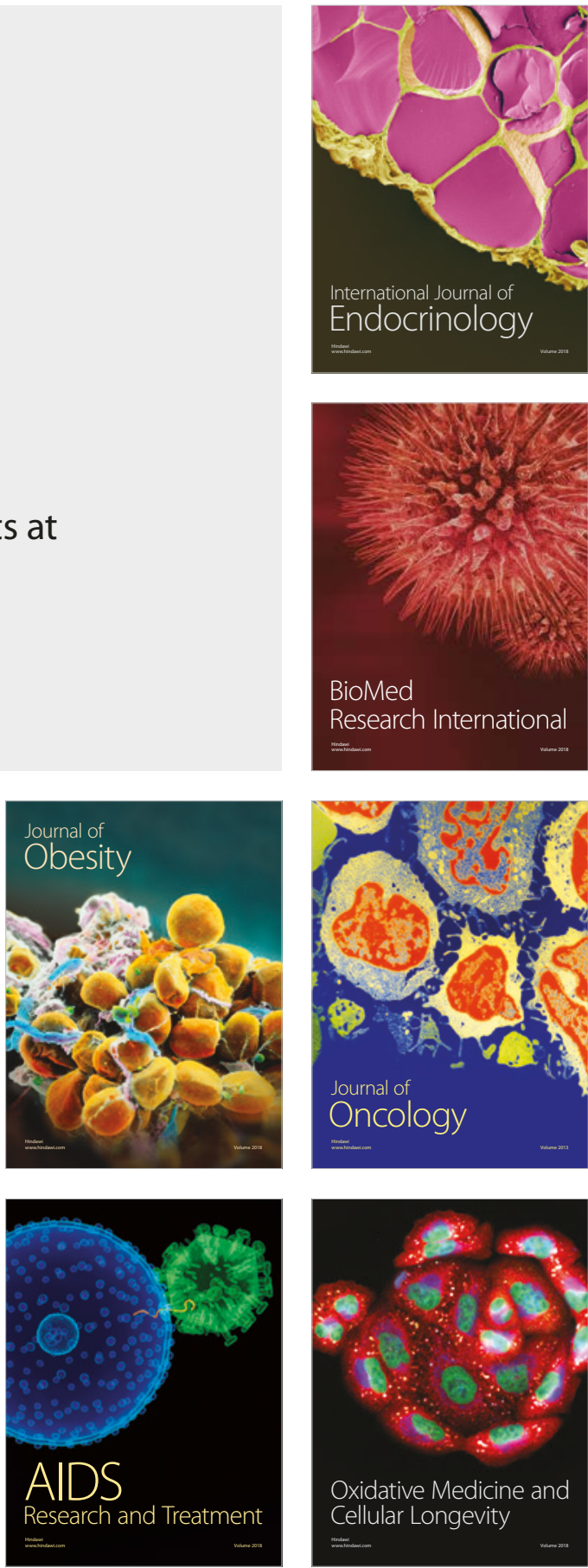